\title{
Mailbox
}

\section{A correction to "Definable principal congruences in varieties of groups and rings"}

S. Burris and J. LAwrence

In the paper cited above [1], the proofs of Theorems 1.2 and 1.3 are incorrect. We do not know if the results, as stated, are correct. The error lies in assuming that for all $\omega(x, y, u, v, \vec{z})$ in $\Gamma$ one can claim

$$
F_{k} \vDash \omega\left(\bar{x}, \bar{y}, \vec{u}, \bar{v}, \bar{z}_{0}, \ldots, \bar{z}_{n}\right)
$$

as at the top of page 154. However for a restricted class of $\omega$ in $\Gamma$ this claim holds, and if one replaces the lemma and theorems of $\S 1$ by the following text then one has a result which is sufficiently strong for the study of groups and rings as in $\S 2,3$. (The main results, those of $\S 2,3$, are correct as presented in [1].)

LEMMA 1. If $K$ is closed under ultra products, then given formulas $\left\{\phi_{i}\right\}_{i \in I}$ and $\phi$, we have $K \vDash \underset{i \in J}{W} \phi_{i} \leftrightarrow \phi$ iff for some finite $J \subseteq I, K \vDash \underset{i \in J}{w} \phi_{i} \leftrightarrow \phi$.

Proof. (Standard.)

DEFINITION 2. Let $P$ be the set of polynomials $p\left(w, z_{0}, \ldots, z_{n}\right), n<\omega$. For $P_{0} \subseteq P$, a variety $V$ has $P_{0}$-projective principal congruences if, for $a, b, c, d \in A \in V$, $(a, b) \in \theta_{A}(c, d)$ holds iff

$$
A \vDash \exists \vec{z}\left[a=p\left(e_{1}, \vec{z}\right) \& b=p\left(e_{2}, \vec{z}\right)\right]
$$

for some $p \in P_{0}$, where $\left\{e_{1}, e_{2}\right\}=\{c, d\}$.

Presented by G. Grätzer. Received April 5, 1979. Accepted for publication in final form March 6, 1980. 
Two examples of varieties with $P_{0}$-projective principal congruences follow:

(1) For rings let $P_{0}=\left\{p_{n}: n \geq 1\right\}$ where

$$
p_{n}\left(w, z_{0}, \ldots, z_{2 n+1}\right)=\sum_{i=0}^{n-1} z_{2 i} \cdot\left(w-z_{2 n}\right) \cdot z_{2 i+1}+z_{2 n+1} .
$$

To see that this works let $a, b, c, d \in R, R$ a ring. Then $(a, b) \in \theta_{R}(c, d)$ iff $a-b$ is in the ideal generated by $c-d$ iff for some $n<\omega$ and some $a_{0}, \ldots, a_{2 n-1}$

$$
a-b=\sum_{i=0}^{n-1} a_{2 i}(c-d) a_{2 i+1}
$$

But then

$$
\begin{aligned}
& a=p_{n}\left(c, a_{o}, \ldots, a_{2 n-1}, d, b\right) \\
& b=p_{n}\left(d, a_{0}, \ldots, a_{2 n-1}, d, b\right) .
\end{aligned}
$$

(2) For groups of finite exponent $e$ let $P_{0}=\left\{p_{n}: n \geq 1\right\}$ where

$$
\left.p_{n}\left(w, z_{0}, \ldots, z_{n+1}\right)=\left[\prod_{i=0}^{n-1} z_{i}^{-1} \cdot\left(w \cdot z_{n}^{-1}\right) \cdot z_{i}\right)\right] \cdot z_{n+1} .
$$

If $G$ is a group of exponent $e$ and $a, b, c, d \in G$ then $(a, b) \in \theta_{G}(c, d)$ iff $a b^{-1}$ is a product of conjugates of $c d^{-1}$, hence iff for some $n$ and $a_{i} \in G$,

$$
a b^{-1}=\prod_{i=0}^{n-1} a_{i}^{-1}\left(c d^{-1}\right) a_{i}
$$

But then

$$
\begin{aligned}
& a=p_{n}\left(c, a_{0}, \ldots, a_{n-1}, d, b\right) \\
& b=p_{n}\left(d, a_{0}, \ldots, a_{n-1}, d, b\right) .
\end{aligned}
$$

THEOREM 3. Let $V$ be a variety with $P_{0}$-projective principal congruences, for a given $P_{0}$. Then $V$ has DPC iff for some finite subset $P_{0}^{\prime}$ of $P_{0}$ there is, for each $p\left(w, z_{0}, \ldots, z_{n}\right) \in P_{0}, a q\left(w, z_{0}, \ldots, z_{k}\right) \in P_{0}^{\prime}$ and polynomials $q_{i}\left(u, v, z_{0}, \ldots, z_{n}\right)$, $0 \leq i \leq k$, such that $V$ satisfies, for suitable $\left\{w_{1}, w_{2}\right\}=\{u, v\}$,

$$
\begin{aligned}
& p\left(u, z_{0}, \ldots, z_{n}\right)=q\left(w_{1}, q_{0}\left(u, v, z_{0}, \ldots, z_{n}\right), \ldots, q_{k}\left(u, v, z_{0}, \ldots, z_{n}\right)\right) \\
& p\left(v, z_{0}, \ldots, z_{n}\right)=q\left(w_{2}, q_{0}\left(u, v, z_{0}, \ldots, z_{n}\right), \ldots, q_{k}\left(u, v, z_{0}, \ldots, z_{n}\right)\right) .
\end{aligned}
$$


Proof. $(\Rightarrow)$ Suppose $V$ has $D P C$. Then from Lemma 1 there must be a $P_{0}^{\prime} \subseteq P_{0}$ such that $V$ satisfies

$$
\begin{aligned}
& \left.\left(^{*}\right) \underset{p \in P_{0}}{\underset{w}{W}} \exists \vec{z}[x=p(u, \vec{z}) \& y=p(v, \vec{z}))\right] \\
& \leftrightarrow \underset{\mathbf{q} \in P_{\mathbf{o}^{\prime}}}{\underset{z}{W}} \exists \bar{z}\left[x=q\left(w_{1}, \vec{z}\right) \& y=q\left(w_{2}, \vec{z}\right)\right] \\
& \left\{w_{1}, w_{2}\right\}=\{u, v\}
\end{aligned}
$$

Given $p\left(w, z_{0}, \ldots, z_{n}\right) \in P_{0}$ let $F$ be the free algebra in $V$ freely generated by $u, v, z_{0}, \ldots, z_{n}$. In $F$ let $x=p\left(u, z_{0}, \ldots, z_{n}\right), y=p\left(v, z_{0}, \ldots, z_{n}\right)$. As

$$
F \vDash \exists \vec{z}[x=p(u, \vec{z}) \& y=p(v, \vec{z})]
$$

it follows by $\left(^{*}\right)$ that for some $q\left(w, z_{0}, \ldots, z_{k}\right) \in P_{0}^{\prime}$,

$$
F \vDash \exists \vec{z}\left[x=q\left(w_{1}, \bar{z}\right) \& y=q\left(w_{2}, \bar{z}\right)\right]
$$

with $\left\{w_{1}, w_{2}\right\}=\{u, v\}$. Thus we can choose polynomials $q_{i}\left(u, v, z_{0}, \ldots, z_{n}\right) \in F$ such that

$$
\begin{aligned}
& F \vDash x=q\left(w_{1}, q_{0}\left(u, v, z_{0}, \ldots, z_{n}\right), \ldots, q_{k}\left(u, v, z_{0}, \ldots, z_{n}\right)\right) . \\
& F \vDash y=q\left(w_{2}, q_{0}\left(u, v, z_{0}, \ldots, z_{n}\right), \ldots, q_{k}\left(u, v, z_{0}, \ldots, z_{n}\right)\right) .
\end{aligned}
$$

Of course if two polynomials are equal in $F$ then the corresponding identity holds in $V$.

$(\Leftarrow)$ Let $a, b, c, d \in A \in V \quad$ with $(a, b) \in \theta_{A}(c, d)$. Then, for some $p\left(x, z_{0}, \ldots, z_{n}\right) \in P_{0}$,

$$
A \vDash \exists \bar{z}\left[a=p\left(e_{1}, \vec{z}\right) \& b=p\left(e_{2}, \vec{z}\right)\right]
$$

with $\left\{e_{1}, e_{2}\right\}=\{c, d\}$. Choose $q, q_{0}, \ldots, q_{k}$ as in the statement of the theorem. Then, for suitable $\left\{\bar{e}_{1}, \bar{e}_{2}\right\}=\{c, d\}$,

$$
\begin{aligned}
& A \vDash \exists \vec{z}\left[a=q\left(\bar{e}_{1}, q_{0}\left(\bar{e}_{1}, \bar{e}_{2}, \vec{z}\right), \ldots, q_{k}\left(\bar{e}_{1}, \bar{e}_{1}, \vec{z}\right)\right)\right. \\
& \left.\& b=q\left(\bar{e}_{2}, q_{0}\left(\bar{e}_{1}, \bar{e}_{2}, \vec{z}\right), \ldots, q_{k}\left(\bar{e}_{1}, \bar{e}_{2}, \vec{z}\right)\right)\right]
\end{aligned}
$$


so

$$
A \vDash \exists \bar{z}\left[a=q\left(\bar{e}_{1}, \vec{z}\right) \& b=q\left(\bar{e}_{2}, \vec{z}\right)\right] .
$$

Thus the formula $\phi(x, y, u, v)$ given by

$$
\underset{\substack{q \in P_{0}^{\prime} \\\left\{w_{1}, w_{2}\right\}=\{u, v\}}}{\bigvee} \exists \bar{z}\left[x=q\left(w_{1}, \vec{z}\right) \& y=q\left(w_{2}, \vec{z}\right)\right]
$$

defines principal congruences in $V$.

COROLLARY 4. Let $V(K)$ be a variety with $P_{0}$-projective principal congruences. Then $V(K)$ has $D P C$ iff $Q(K)$ has $D P C$.

Proof. The direction $(\Rightarrow)$ is clear. For $(\Leftrightarrow)$ just repeat the first part of the proof of Theorem 3 as $F \in Q(K)$.

PROBLEM: For arbitrary $K$ is it true that $Q(K)$ has $D P C$ implies $\mathbf{V}(K)$ has $D P C$ ?

\section{REFERENCES}

[1] S. BURRIS and J. LAWRENCE, Definable principal congruences in varieties of groups and rings. Alg. Univ. 9 (1979), 152-164.

University of Waterloo Waterloo, Ontario

Canada 